\title{
Management of Tubercular Pyonephrosis: A Single Centre Experience from Northern India
}

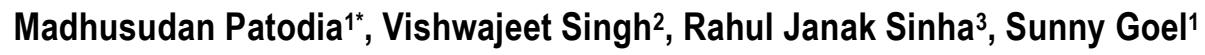 \\ ${ }^{1}$ Senior resident, ${ }^{2}$ Professor, ${ }^{3}$ Associate Professor, \\ Department of Urology, King George Medical University, Lucknow, Uttar Pradesh INDIA.
}

\begin{abstract}
Objective: To analyze the clinical presentation and outcome of tubercular pyonephrosis from single centre in northern India.

Methods: Patients with diagnosis of tubercular pyonephrosis managed at our institution from July 2004 to December 2015 were retrospectively analyzed. Data regarding history, physical examination, routine blood investigation, urine/pus examination, X-ray, ultrasound, intravenous urogram, polymerase chain reaction, micturating cystourethrogram, cysto-urethroscopy, retrograde pyelogram, computed tomography scan and Tc-99m diethylene-triamine-penta-acetic acid renal scan were recorded.

Results: Total 28 patients were included in the study diagnosed as tubercular pyonephrosis on histopathological examination. Total leukocyte count was raised in 13 (46.42\%) patients, and ESR was raised in $12(42.85 \%)$ patients. On urine examination, acid fast bacilli's staining was positive in 7 $(25 \%)$ patients. Urine culture was positive for mycobacterium in $9(32.14 \%)$ patients and positive for other bacteria in 9 $(32.14 \%)$ patients. Mauntoux test was positive in $8(28.57 \%)$ patients. On ultrasonography, four patients had hydronephrosis, six patients had small kidney and 18 patients had pyonephrosis. On plain X-ray, 7 (25\%) patients had positive findings suggestive of tuberculosis. Intravenous urography was suggestive of tubercular pathology in 9 $(32.14 \%)$ patients. CECT scan was performed in 22 patients and given evidence of tubercular pathology in 11 patients. RGP was performed in 10 patients and showed ureteric involvement
\end{abstract}

\section{INTRODUCTION}

Genitourinary tuberculosis (GUTB) is reported to be the third most common site of tuberculosis, after pleural and lymphatic. ${ }^{1}$ It occurs by hematologic route and represents an earlier pulmonary tubercular infection which has remained dormant for many years. ${ }^{1}$ It is still highly prevalent in developing countries although incidence has drastically reduced in developed nations. ${ }^{2}$ Around $50 \%$ of India's population above the age of 20 years is infected with tubercular bacilli. ${ }^{3}$ The kidney is the most site of genitourinary tuberculosis.

The diagnosis of GUTB is difficult due to non-specific signs and symptoms. This often delays the diagnosis and ultimately leading to pyonephrosis. In our study, we have retrospectively analyzed the clinical features, diagnostic tests, surgeries performed and pathological findings of tubercular pyonephrosis (TPN). Here, we discuss how the clinical presentation of tubercular pyonephrosis in last one decade at our centre.

\section{MATERIAL AND METHODS}

We analysed the case records of patients with diagnosis of in 6 patients. Two patients found to have small capacity bladder on micturating cystourethrogram and these findings were confirmed on cystoscopy. After radiological and endoscopic evaluation, we were able to make preoperative diagnosis of renal tuberculosis in only $17(60.71 \%)$ patients.

Conclusion: Clinical presentation of tubercular pyonephrosis varies from subclinical infection to fulminant form. Various radiological modalities cannot diagnose all cases preoperatively. The histopathological examination can diagnose all cases post-operatively.

Keywords: Genito-urinary tuberculosis, Pyonephrosis, Renal tuberculosis, Tubercular pyonephrosis.

\section{*Correspondence to:}

Madhusudan Patodia, Senior resident, Urology, KGMU, Lucknow, Uttar Pradesh, INDIA.

Email: madhusudanpatodia@gmail.com

Article History:

Received: 13-04-2016, Revised: 27-04-2016, Accepted: 14-05-2016

\begin{tabular}{|l|c|}
\hline \multicolumn{2}{|c|}{ Access this article online } \\
\hline Website: & Quick Response code \\
www.jimrp.com & \\
\hline DOI: & \\
10.21276/ijmrp.2016.2.3.033 & \\
\hline
\end{tabular}

tubercular pyonephrosis managed at our institution from July 2004 to December 2015. Evaluation included comprehensive history, physical examination, routine blood investigation, urine/pus examination/culture (bacterial and mycobacterium), X-ray chest, X-ray KUB, ultrasound KUB, and intravenous urogram (IVU). Findings of polymerase chain reaction (PCR) for detecting deoxyribonucleic acid (DNA) of mycobacterium tuberculosis, micturating cystourethrogram (MCU), cysto-urethroscopy, retrograde pyelogram (RGP), contrast enhanced computed tomography (CECT) scan whole abdomen and Tc-99m diethylene-triamine-penta-acetic acid (DTPA) renal scan were also recorded.

\section{Inclusion criteria}

Patients managed by nephrectomy/nephroureterectomy with diagnosis of tubercular pyonephrosis on histopathology.

\section{Exclusion criteria}

Patients treated conservatively and with non-tubercular histopathology. 
Previous history of tuberculosis/treatment was considered when patient had taken treatment for tuberculosis in past (defaulter/ resistant/ recurrence / failure patients also included).

Positive family history was considered when family member who is living with patient in the same house had history of tuberculosis. Malnutrition was defined as (body mass index) $\mathrm{BMl}<18.5 \mathrm{~kg} / \mathrm{m}^{2}$. Haemoglobin was considered normal if $\mathrm{Hb}>10 \mathrm{~g} / \mathrm{dl}$. Three consecutive urine samples were examined for urine/pus culture for bacteria and mycobacteria.

Our institutional protocol is to confirm the diagnosis of pyonephrosis and placement of percutaneous nephrostomy (PCN) tube followed by anti-tubercular treatment (ATT) and culture sensitive antibiotics. If differential renal function is found to be less than $10 \%$, nephrectomy or nephroureterectomy was performed after 4 week of anti-tubercular treatment (ATT) and completed the 6 month course of ATT after surgery. If reconstructive surgery is planned then ATT therapy was given for 6 weeks before surgery.
Table 1: Demographic and clinical characteristics of patients

\begin{tabular}{lc}
\hline Variables & $\mathrm{n}=\mathbf{2 8}$ \\
\hline Male : Female & $16: 12$ \\
Mean age & 36.8 \\
Previous history of tuberculosis/treatment & 9 \\
Positive family history & 8 \\
Low income group & 23 \\
Symptoms & \\
Flank pain & 17 \\
Fever & 12 \\
Malaise & 11 \\
Irritative voiding symptoms & 9 \\
Hematuria & 4 \\
History of Diabetes Mellitus & 2 \\
History of COPD & 4 \\
History of HTN & 3 \\
Poor nutritional status & 15 \\
Mean BMI & 20.2 \\
\hline COPD: Chronic obstructive pulmonary disease, HTN: Hypertension, BMl: Body \\
mass index
\end{tabular}

Table 2: Data including laboratory investigations

\begin{tabular}{|c|c|c|}
\hline Variables & $\begin{array}{c}\text { Number of patients with } \\
\text { normal range }\end{array}$ & $\begin{array}{c}\text { Number of patients with positive } \\
\text { findings }\end{array}$ \\
\hline $\mathrm{Hb}(\mathrm{g} / \mathrm{dl})$ & $12(>10 \mathrm{~g} / \mathrm{dl})$ & $16(<10 \mathrm{~g} / \mathrm{dl})$ \\
\hline TLC (4000-11000/cmm) & 15 & $13(>11000 / \mathrm{cmm})$ \\
\hline Platelet count (1.5 lac/cmm) & 25 & $3(<1,50000 / \mathrm{cmm})$ \\
\hline ESR (<30 in $1^{\text {st }}$ hour) & 16 & 12 (Raised) \\
\hline Blood Urea (<20 mg/dl) & 22 & 6 (raised) \\
\hline S. creatinine $(0.5-1.0 \mathrm{mg} / \mathrm{dl})$ & 24 & 4 (raised) \\
\hline \multicolumn{3}{|l|}{ Urinalysis } \\
\hline Reaction $\mathrm{pH}$ & 21 (acidic) & 7 (alkaline) \\
\hline Proteins (<150 mg/24 hours) & 22 & $6(>150 \mathrm{mg})$ \\
\hline Pus cells & 15 (absent) & 13 (present) \\
\hline Erythrocyte & 21 (absent) & 7 (present) \\
\hline Urine/pus for AFB staining & 21 (absent) & 7 (present) \\
\hline Urine/Pus for mycobacterial culture & 19 (absent) & 9 (present) \\
\hline Urine/Pus for bacterial culture & 19 (sterile) & 9 (positive) \\
\hline Anti-HIV antibody & 26 (absent) & 2 (present) \\
\hline HbsAg antigen & 26 (absent) & 2 (present) \\
\hline Anti-HCV antibody & 27 (absent) & 1 (present) \\
\hline Mauntoux Test (>15 mm) & 20 (negative) & 8 (positive) \\
\hline TB PCR & 14 (negative) & 14 (positive) \\
\hline
\end{tabular}

Hb: Haemoglobin, TLC: Total leukocyte count, ESR: Erythrocyte sedimentation rate, AFB: Acid fast bacilli, TB PCR: Tuberculosis polymerase chain reaction

Table 3: Data regarding radiological investigations

\begin{tabular}{lcc}
\hline Investigation & $\begin{array}{c}\text { Number of patients with evidence of renal, } \\
\text { ureteric and bladder involvement in GUTB }\end{array}$ & $\begin{array}{c}\text { Number of patients with no } \\
\text { evidence of GUTB }\end{array}$ \\
\hline Renal ultrasonography & $\begin{array}{c}\text { Hydronephrosis in 4 patients } \\
\text { Pyonephrosis in 18 patients } \\
\text { Small kidney in } 6 \text { patients }\end{array}$ \\
$\begin{array}{l}\text { X-Ray KUB and Chest X-Ray PA view } \\
\text { IVU (Performed in 20 patients) }\end{array}$ & 7 & 21 \\
CECT scan (Performed in 22 patients) & 9 & 11 \\
MCU (Performed in 9 patients) & 11 & 11 \\
RGP (Performed in 10 case) & & \\
Renal scan (Performed in 15 patients) & & \\
CPE (Performed in 9 patients) & Small capacity bladder in 2 patients & \\
& Ureter involved in 6 patients & \\
& NFK/very poor function in 14 patients & \\
\hline
\end{tabular}

IVU: Intravenous urogram, CECT scan: Contrast enhanced computed tomography scan, MCU: Micturating cystourethrogram, RGP: Retrograde pyelogram, CPE: Cysto-pan-endoscopy

Table 4: Data regarding treatment of patients

\begin{tabular}{lc}
\hline Management & $\mathrm{n}=\mathbf{2 8}$ \\
\hline Nephrectomy & 20 \\
Nephroureterectomy & 6 \\
Nephroureterectomy + Augmentation cystoplasty & 2 \\
\hline
\end{tabular}

\section{RESULTS}

Total 28 patients were included in the study diagnosed as tubercular pyonephrosis on histopathological examination. It is a disease of middle age as mean age was 36.8 years in the study group. Males predominantly affected (ratio males to females was 16:12). Nine $(32.14 \%)$ patients had previous history of 
tuberculosis and eight (28.57\%) patients had positive family history which is common in India. Twenty one $(75 \%)$ patients lived with a combined family and $23(82.14 \%)$ patients belong to low income group. Tuberculosis is a disease of poor people and occurred more commonly in crowded areas. The most common symptom was flank pain $(60.71 \%)$. In most of patients flank pain was associated with fever (42.85\%) and malaise (39.28\%). This is presenting scenario of pyonephrotic kidney. Nine patients presented with history of lower urinary tract symptoms (LUTS). On further evaluation only 2 patients found to have bladder involved simultaneously with kidney involvement.

On routine blood investigation total leukocyte count was raised in $13(46.42 \%)$ patients. Erythrocyte sedimentation rate (ESR) was raised in $12(42.85 \%)$ patients. On urine examination only $7(25 \%)$ patients had acid fast bacilli staining positive and 9 (32.14\%) patients had mycobacterial culture positive after culture of three consecutive samples. Mauntoux test was positive in $8(28.57 \%)$ patients. Only 10 (35.71\%) patients can be diagnosed after blood and urine test. On ultrasonography (USG) four patients had hydronephrosis, 18 patients had pyonephrosis with decreased cortical thickness and 6 patients had small contracted kidney. In three patients chest $X$-ray showed positive findings and in five patients X-ray KUB showed calcification in KUB area (Total seven (25\%) patients had positive findings on plain $X$-ray, as one patient had findings on both chest X-ray and X-ray KUB). IVU was performed in 20 patients and suggestive of tubercular pathology in $9(45 \%)$ patients. CECT scan was performed in 22 patients (8 patients in whom IVU was not performed, 11 patients in which IVU was non-diagnostic, 3 patients in which IVU had diagnosis of TPN) and given evidence of tubercular pathology in 11 (50\%) patients. With the help of CECT scan, diagnosis of TPN was made in 5 patients in whom IVU was not performed, 3 patients in whom IVU was non-diagnostic. Total 17 patients were diagnosed preoperatively after radiological imaging. DTPA scan was performed in 15 patients and revealed nonfunctioning kidney in 14 patients. MCU was performed in nine patients who had history of LUTS. Two patients found to have small capacity bladder and these findings were also found on cysto-urethroscopy. The bladder had evidence of chronic cystitis in nine patients on cysto-urethroscopy. Bladder biopsy was diagnostic of TB in one out of two patients. RGP was performed in 10 patients and showed ureteric involvement 6 patients.

We placed PCN in 14 pyonephrotic kidneys and 2 patients of hydronephrosis which were having fever. Pus was sent for mycobacterial and bacterial culture and sensitivity. Patients received antitubercular therapy (ATT) for a total of 6-12 months. Twenty patients underwent open nephrectomy. Six patients underwent nephroureterectomy and two patients underwent nephroureterectomy with augmentation cystoplasty.

\section{DISCUSSION}

Urogenital system constitutes one of the most common sites of extra-pulmonary tuberculosis. ${ }^{4}$ Every day, around 1000 deaths are reported in India due to tuberculosis. ${ }^{5}$

Renal tuberculosis occurs as a result of reactivation of tubercular bacilli after a prolonged period of latency, or may occur due to reinfection from active tuberculosis. ${ }^{6}$ Tubercular pyonephrosis may involve the kidney as a localized disease or can occur as a generalized disseminated infection. Tubercular bacilli affect the kidney by causing ulceration, cavitation and fistulization. Host's response to tubercular bacilli can cause granulomas fibrosis ultimately leading to stricture formation.

Pathologically, tubercular pyonephrosis initially leads to formation of cortical granulomas which gradually enlarge and coalesce. These granulomas may heal spontaneously, or remain stable in some individuals. ${ }^{7}$ These granulomas are small in size $(<3 \mathrm{~mm}$ in diameter), hence can be missed. ${ }^{8}$ Granulomas later spread to ureters and bladder. The ensuing infection ultimately leads to strictures and pelvicalyceal system dilatation. The combination of obstruction and ongoing inflammation leads to destruction of kidneys. Patients usually present with frequency, pyuria, hematuria and decreasing renal function. Involvement of bladder leads to lower urinary tract symptoms. Tuberculosis may lead to unexplained urological symptoms. Patients not responding to antibiotics, having sterile pyuria on urine examination lead to suspicion of tuberculosis. ${ }^{9}$

Tubercular pyonephrosis typically has an insidious onset with nonspecific symptoms which leads to delay in diagnosis. Most of our patients presented with fever, flank pain and malaise. Most of the patients presented late in advanced stage of pyonephrosis leading to destruction of whole of the kidney. This is because of poverty, poor medical infrastructure and lack of awareness towards health in rural areas. Many of the patients had history of antibiobic (Quinolones) abuse, hence leading to symptom relief and presenting late. Irrational and liberal use by local medical practitioners also leads to emergence of antibiotic resistance. In last, many patients had history of tuberculosis in past with incomplete course of ATT intake.

Laboratory investigations revealed raised total leukocyte count in 13 patients and raised ESR in 12 patients. About $50 \%$ of patients had findings suggestive of chronic infection. In TPN patients, tubercular bacilli usually spread hematogenously, lodged in glomerular and peritubular capillary bed leading to formation of granulomas. The granulomas either heal spontaneously or may rupture into renal tubules or the interstitium. The intermittent rupture may lead to absence of pyuria, hematuria or AFB in urine culture. Because of this, many patients show normal urinary findings. Microscopy with Ziehl Neilsen staining can reveal AFB in $72 \%$ of patients. ${ }^{6}$ In our study, AFB could be detected in only 7 patients $(25 \%)$ indicating low sensitivity of the test. Three serial urine cultures were taken for detection of active tuberculosis. ${ }^{10}$ Adhering to this regime also failed to grow AFB in 19 (67.85\%) of the patients. It may be presence of chronic renal lesions that no longer discharge tuberculous material in urine due to formation of dense fibrosis that acts as a barrier to the pelvicalyceal system. Acquired cellular immunity may inhibit bacterial multiplication leading to containment of disease in many patients; culminating in formation of granulomas. ${ }^{11}$ Administration of antitubercular chemotherapy for active focus may also result in healing. Incomplete and/or intermittent treatment by primary care physicians for tuberculosis also leads to low detection rate.

Polymerase chain reaction (PCR) was positive in 14 (50\%) patients. The sensitivity of PCR is $87 \%$ to $95 \%$ and specificity is $92 \%$ to $99.8 \%$ compared to urine culture. Presence of naturally occurring enzyme inhibitors in up to $10 \%$ of patients usually suppresses enzymatic reactions of DNA/RNA amplification. This leads to increase in false-negative results. This may be the reason for low detection rate by PCR in our study. 
Imaging including ultrasonography, computerized tomography demonstrates hydronephrosis, distorted ureters with alternating stenotic and dilated areas, reduced bladder volume and small kidneys. ${ }^{12,13}$ The role of ultrasonography is limited because of nonspecific findings. It is used for follow up. Although findings on CT are also non-specific, the collective evidence on imaging and clinical picture helps in decision making.

Intravenous urography shows calyceal distortion and decline of kidney function $[12,14]$. Our patients which had positive findings on X-ray were $7(25 \%)$. Total $9(45 \%)$ patients have positive findings on IVU out of 20 patients in which IVU was done. CECT scan was not able to diagnose 11 patients. Patients who had positive findings on MCU, RGP and CPE, also had findings in IVU or CECT scan, so in our study MCU, RGP and CPE were not able to make diagnosis in a single case. However these investigations were helpful in planning of surgery. In these $11(39.28 \%)$ patients, we were not able to diagnose the disease preoperatively. These 11 patients were diagnosed as tubercular pyonephrosis after histopathology of nephrectomy specimen. These are patients who presented to us with pyonephrosis and managed with initial nephrostomy placement. Pus culture in these patients was negative for tubercular bacilli. All of these patients underwent nephrectomy and intra-operatively there were dense adhesions surrounding the kidney. In patients of renal parenchymal involvement with persistence pus discharge or in patients where the kidney remains nonfunctioning despite adequate drainage it would be better to perform a nephrectomy to remove of the infective pathology.

Unlike tuberculosis of other organs, $\geq 50 \%$ of the patients with TPN needed surgical treatments. Anti-tuberculous treatment must be combined with surgery in patients of TPN. Open nephrectomy was performed in all cases.

Tuberculosis is still widely prevalent and a matter of concern because of poverty, increasing incidence of HIV patients and large migration of people. Extrapulmonary TB commonly occurs in patient of organ transplant and HIV. Around 1/3 of HIV patients are co-infected with tuberculosis. Reasons for acquired resistance to anti-tubercular therapy include inadequate treatment, lack of compliance and low quality medicines. Five percent of patients do not respond to isoniazid and rifampicin. Defaulters promote the emergence of resistant tubercular strains. Multidrug resistant forms leads to fulminant form of the disease, whose treatment is difficult, costly and lethal.

\section{CONCLUSION}

Clinical and pathological presentation of renal tuberculosis is changing in recent times. Renal tuberculosis is presenting as more fulminant form in some patients and at the same time subclinical form in other group of patients. Nephrectomy is the preferred treatment for management along with complete antitubercular treatment in such patients. Some of the patients might require additional treatment in the form of augmentation cystoplasty to save the remaining kidney.

\section{ETHICAL APPROVAL}

All procedures performed in studies involving human participants were in accordance with the ethical standards of the institutional and/or national research committee and with the 1964 Helsinki declaration and its later amendments or comparable ethical standards.

\section{INFORMED CONSENT}

Informed consent was obtained from all individual participants included in the study.

\section{REFERENCES}

1. Kennedy DH. Extrapulmonary tuberculosis. In: Ratledge C, Stanford JL, Grange JM, editors. The Biology of Mycobacteria. New York: Academic Press; 1989. pp. 245-284.

2. Sharma SK, Mohan A. Extrapulmonary tuberculosis. Indian J Med Res. 2004;120:316-53.

3. George Tharayal John, Viswanathan Shanker, Abi Mookanottle Abraham, Uma Mukandan, Paulose Pannakuzhathil Thomas, Chakko Korula Jacob, risk factor for post-transplant tuberculosis: Kidney International: 2001: 60:1148 - 1153.

4. Wise GJ, Marella VK. Genitourinary manifestations of tuberculosis. Urol Clin North Am. 2003;30:111-21.

5. Chauhan LS, Tonsing J. Revised national TB control programme in India. Tuberculosis (Edinb) 2005;85:271-6.

6. AJ Harding, H David Ritchie, Bailey and Love's short practice of surgery: 19th Ed: English language book society, Champman and Hall London: 1997: 934 - 936.

7. Birnbaum BA, Friedman JP, Lubat E, Megibow AJ, Bosniak MA. Extrarenal genitourinary tuberculosis: CT appearance of calcified pipestem ureter and seminal vesicle abscess. J Comput Assist Tomogr. 1990;14:653-5.

8. Rook GA. The role of vitamin D in tuberculosis. Am Rev Respir Dis. 1988;138:768-70

9. Eastwood JB, Corbishley CM, Grange JM. Tuberculosis and the kidney. J Am Soc Nephrol. 2001; 12:1307-14.

10. Diagnostic standards and classification of tuberculosis in adults and children. Am J Respir Crit Care Med. 2000; 161:1376-95.

11. Hartman DS, Stagg PL. Diagnosis please. Case 3: Renal tuberculosis. Radiology. 1998;209:69-72.

12. Daher Ede F, Silva Junior GB, Damasceno RT, Santos GM, Corsino GA, Silva SL, Gutierrez-Adrianzen OA, 2007. End-stage renal disease due to delayed diagnosis of renal tuberculosis: a fatal case report. Braz J Infect Dis 11: 169-171.

13. Figueiredo AA, Lucon AM, Arvellos AN, Ramos CO, Toledo AC, Falci R Jr, Gomes CM, Recaverren FE, Netto JM, Srougi M, 2010. A better understanding of urogenital tuberculosis pathophysiology based on radiological findings. Eur J Radiol 76: 246-257.

14. Oliveira JL, Silva Junior GB, Daher EF, 2011. Tuberculosisassociated chronic kidney disease. Am J Trop Med Hyg 84: 843-844

\section{Source of Support: Nil.}

Conflict of Interest: None Declared.

Copyright: (c) the author(s) and publisher. IJMRP is an official publication of Ibn Sina Academy of Medieval Medicine \& Sciences, registered in 2001 under Indian Trusts Act, 1882.

This is an open access article distributed under the terms of the Creative Commons Attribution Non-commercial License, which permits unrestricted non-commercial use, distribution, and reproduction in any medium, provided the original work is properly cited.

Cite this article as: Madhusudan Patodia, Vishwajeet Singh, Rahul Janak Sinha, Sunny Goel. Management of Tubercular Pyonephrosis: A Single Centre Experience from Northern India. Int J Med Res Prof. 2016; 2(3):151-54. 